\title{
Research and Development on Supply Chain Collaboration Management System
}

\author{
Yong Cen \\ China Tobacco Zhejiang Industrial Co., Ltd \\ Hangzhou, 310009 , PR China \\ ceny@zjtobacco.com
}

\begin{abstract}
The fierce competition pressures on supply chain management of tobacco industry enterprise increases emphasis on supply chain agility, integration, and visibility to respond rapidly and efficiently to changes in the marketplace. In this paper, we analysis the requirements and characteristics of the system, then develop the supply chain collaboration management system. This system use S AP XI integration bus technology as the Enterprise Service Bus to tight integration with ERP, PDM and Batch Management System to build a collaborative supply chain management environment in China tobacco industry enterprise. Therefore, this system can realize the sharing, obtain and transfer the various aspects of information of supply chain and to provide insights for the supply chain leading to long term success.
\end{abstract}

Keywords-Supply chain; Collaboration management system; Tobacco industry enterprise

\section{INTRODUCTION}

Supply chain collaboration refers to coordination and joint efforts among partners in a supply chain to improve its overall competitiveness [1]. In an environment of globalization and growing market competition, it has been agreed upon among enterprises in the supply chain that it is high time they strengthen collaboration in order to achieve collaborative operation and management. Within a supply chain setting, collaboration involves two or more independent companies working together to achieve greater success than can be attained in isolation [2]. It is widely accepted that creating collaboration enhances responsiveness, customer service, inventory management, efficient use of resources, and information sharing [3]. Research on supply chain collaboration identified information sharing as one of the common attributes that make supply chain collaboration process successful [4]. The value of demand information sharing between supply chain members and how and under what circumstances that the supply chain member could benefit greatly [5-6]. The buyer seller correlation in order, inventory, and demand information sharing through multiagent simulation system and indicated that such an information sharing could reduce the demand uncertainty [7].

Tobacco industry enterprise supply chain collaboration means the tobacco industry supply chain on the supply of tobacco materials, tobacco products production and sales process in the form of agreements or organizations to make decision incentive balance its logistics, capital flow and information flow, make sure that the individual decisions of supply chain network coordinate with the overall decision. The significance of supply chain collaboration research and application of information management in tobacco industry enterprise refers to achieve synergy in the supply chain automation, information, order, quickly transfer and effective coordination. Finally, through scientific analysis, to achieve the purpose of reasonable control cost and excavates potential value.

\section{SYSTEM ANALYSIS OF THE SUPPLY CHAIN COLLABORATION MANAGEMENT SYSTEM}

\section{A. Characteristics of the System Construction}

The realization of supply chain collaboration includes internal collaboration and synergies between enterprises. Suppliers collaboration management is a kind of "expand cooperation of mutual partnership, common development, and expand market share, achieve win-win" as the guidance of the enterprise resource acquisition management system engineering. This paper considers that the supply chain collaboration management system in tobacco industry enterprise should have the following feature.

1) Meet the requirements of the tobacco industry management.

With the increasing competition between industrial enterprises, integrated supply chain management has become the business development direction of the tobacco industry enterprise. Meanwhile, procurement and supplier relationship management is the weaknesses of enterprises' informatization.

2) Meet the refined management requirements.

Enterprise through the establishment of information platform to support the supply chain collaborative operating model to achieve the procurement centralized, standardization, so as to satisfy the requirements of refined management in enterprise.

3) Promotion of the competitiveness of the enterprise supply chain.

Information platform to support supply chain collaboration can be achieved with upstream suppliers' longterm close partnership, effective use of supplier resources, improve the quality of supply and reduce procurement and inventory costs, thereby enhancing the overall core competitiveness of the enterprise supply chain.

\section{B. System Requirements of Supply Chain Collaboration Management System}

Supply chain collaborative management from the point of view of the management level, generally considered to consist of three levels, namely the strategic layer coordination, the strategy layer collaboration and technical layer collaboration. 


\section{1) Strategic Layer Collaboration}

The strategic layer collaboration is the introduction of the idea and means of supply chain collaborative management to improve the supply chain from the height of the strategy. It mainly includes cultural value integration, unified development goals, risk sharing, profit sharing, resources coordination, collaborative decision making and unified standards, etc. These can be summarized as the supply chain environment, organization and resource coordination.

\section{2) Strategy Layer Collaboration}

The strategy layer collaboration is the core of supply chain collaboration management research. It mainly related to upstream and downstream enterprises in demand forecasting, production planning, procurement, manufacturing, logistics, inventory, and sales and service collaboration.

\section{3) Technical Layer Collaboration}

It mainly refers to provide real-time interactive sharing and communication platform for supply chain node enterprises by technology approach. The content mainly includes data collection, standardized of storage and transmission, information platform to build and intelligent processing.

The supply chain collaboration framework as the Figure 1 shown:

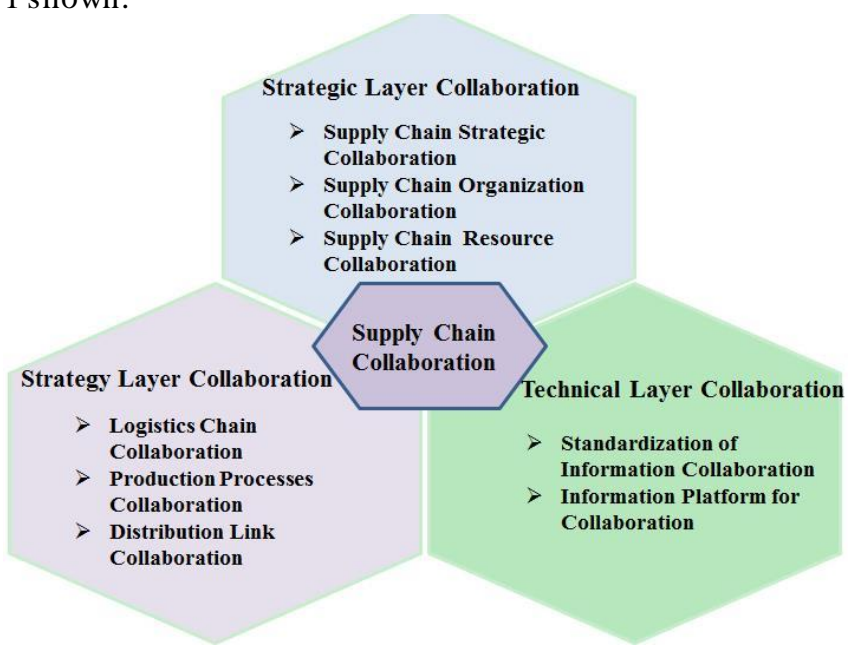

Figure 1. The supply chain collaboration framework

\section{SYSTEM DESIGN AND DEVELOPMENT}

\section{A. System Functions Design}

In the tobacco industry enterprise material supply chain management, supply chain collaboration between suppliers and enterprise, need to have both pushes and pull of network information interaction characteristics. The focus is to strengthen every link of logistics information flow management and control in real time. According to the characteristics of enterprise business and information system construction requirements, the system main functions can be divided into three parts as follows:

\section{1) Strategic Management}

- Suppliers Management: According to the tobacco industry management requirements, purchasing department using a variety of forms such as bidding and for quotation to help company to find tobacco materials suppliers. Those supplier which enter the purchasing business scope, must carry out the corresponding qualification certification.

- Framework Agreements: In the system, procurement framework agree ments signed with various suppliers, and to monitor progress in the implementation of procurement executive the in accordance with the framework agreement.

- Suppliers Evaluation: In the system to establish scientific and perfect supplier performance evaluation system, to maintain the corresponding evaluation rules, set corresponding different depending on the type of supplier assessment scheme and assessment frequency. Conduct a comprehensive assessment of suppliers from the cost of procurement, supplier quality, delivery, service timeliness evaluation index. Set for different types of suppliers cost, quality, productivity, research and development, service and other categories of indicators, according to the tobacco industry related standard, the supplier of these indicators compared with standard value, make similar suppliers find the gap between different indicators.

2) Program Management

- Procurement Plan: According to the annual and monthly Material Requirement Planning (MRP), to make the annual and monthly material procurement plan.

- Preparation Plan: Each supplier based on annual and monthly procurement plan, formu late corresponding monthly stocking planning and production scheduling plan in the system.

\section{3) Execution Management}

- Contracts Management: According to the monthly purchase order in ERP system, sign a contract with suppliers in the system.

- Real-time Inventory Management: Suppliers to fill in the finished goods and work-in-progress real-time inventory change information in the system. System with warehouse physical inventory, in-transit inventory combine to form a safety stock warning prompts, suggesting that buyers place orders.

- Quality Management: When occurrence quality problems, suppliers in the system processing the Quality Notification information created by the ERP system, finally, the results will be passed to the ERP system.

\section{B. System Architecture Design}

The system frame design according the business characteristics and enterprise information system integration demand, system structure is divided into the Presentation 
Layer, Application Service Layer and Data Layer, that structure is shown as the Figure 2:

\section{1) Presentation Layer}

Supply chain management information system terminal according to authority information and the browser to realize information input and browse. In the Web server for identity authentication, then use Hypertext Transfer Protocol transfer the information to internal users and suppliers' users.

\section{2) Application Service Layer}

Application service layer mainly provide all kinds of business and system application services. The layer on the one hand functions responsible for the user's demand response and feedback from the database to get results, and on the other hand, complete web server various kinds of data exchange between infrastructure layers.

\section{3) Data Layer}

The data layer includes data access and integration, network, operation system, etc. This layer mainly manages data access, deal and database management system.

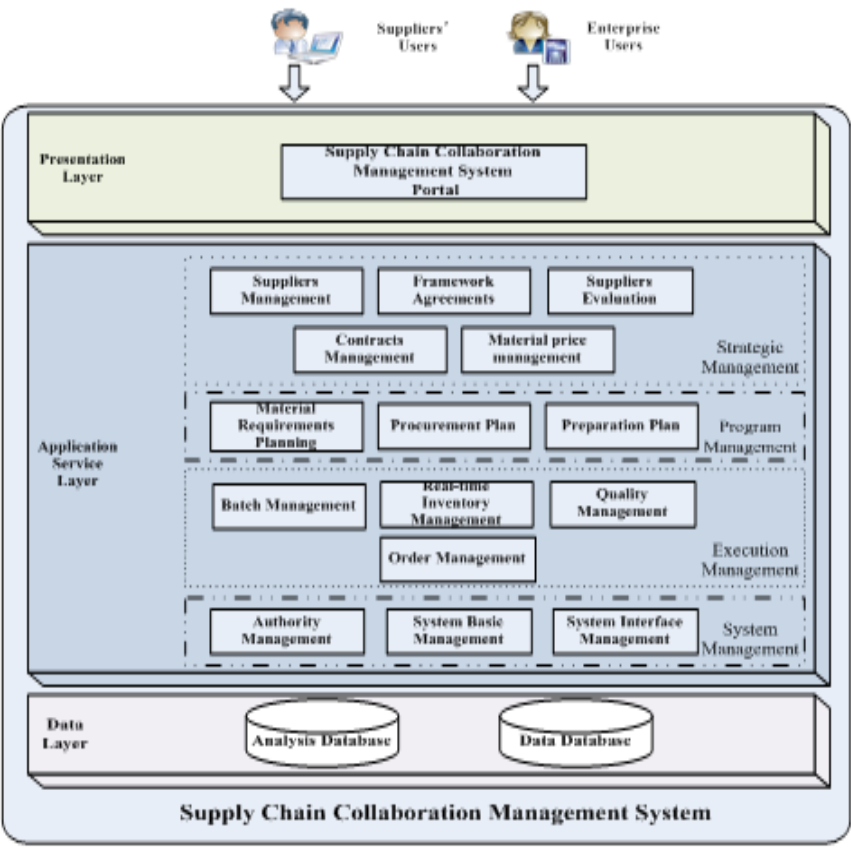

Figure 2. The supply chain collaboration framework

\section{Integration with Systems in Enterprise}

To achieve information and business collaborative computing, a variety of computing resources are integrated in a supply chain. However, the various computing resources to be collaborated in a supply chain differ vastly in physics and logics. In addition, a supply chain system is a dynamic enterprise alliance, and computing resources which participate in collaborative computing also possess certain degree of dynamics. From the above, it can be seen that the main is sue facing technical collaboration in a supply chain is how to effectively integrate distributed computing resources that are dynamically different to achieve collaborative computing and to support new collaborative computing application demand. And this is also a key issue, if we want to effectively enhance overall competitiveness in the supply chain and to improve customer satisfaction.

Therefore, based on the construction of the status quo of enterprise application system and combined with features of SAP XI (Exchange Infrastructure), use the SAP XI as the Enterprise Service Bus (ESB), through the SOAP protocol and star-shape structure to realize the supply chain collaboration system integrated with various heterogeneous systems, such as ERP, PDM and Batch Management System (BMS) etc. The system integration as the Figure 3 shown:

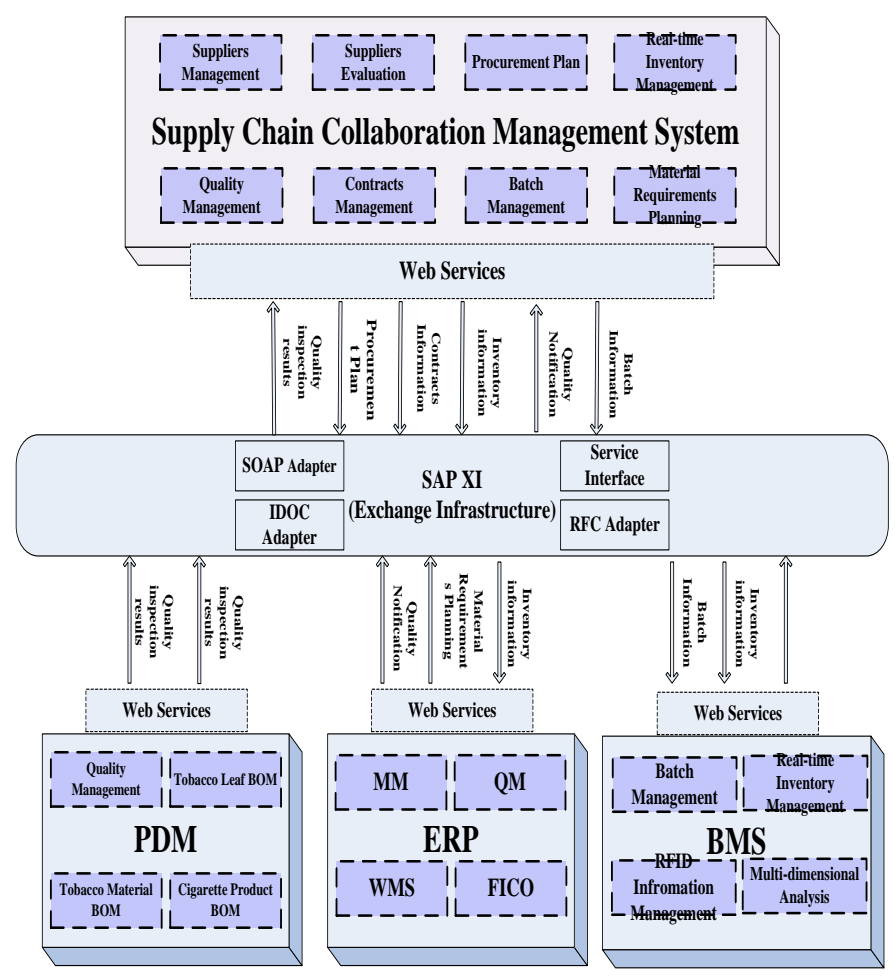

Figure 3. The supply chain collaboration framework

\section{CONCLUSIONS}

Nowadays, automatic identification technologies, network infrastructures, and electronic data exchange technologies have revamped the supply chain landscape. For the development under the pressure of fierce competition, it is critical for enterprise to reorganize and optimize their supply chain effectively, by taking a holistic view of the supply chain. The supply chain integration bridges information gaps and allows effective and efficient information sharing within the supply chain network. This paper presents a supply chain collaboration management system and using the SAP XI technology to integrate with enterprise core systems, such as ERP, PDM and BMS. The proposed system useful to manage the supply chain complexity, exploit network opportunities in the turbulent market environment. And the last, the supply chain collaboration management system will enhance and improve 
the competitive advantage of the enterprise under fluctuating market.

\section{REFERENCES}

[1] Manthou V, V lachopoulouM, Folinas D. Virtuale Chain (VeC) model for supply chain collaboration [J]. Int. J. Production Economics, 2004, 87:241 250.

[2] Bei Zhao, Zhenghui YuanSupply Chain Collaboration: the Mediating Role bet ween Embeddedness and Corporate Performance.

[3] Huiping Lin, Xuwei Zhu, Pengfer Wang, et al. A Service Oriented Supply Chain Collaboration System for SMEs. 2011 6th IEEE Conference on Industrial Electronics and Applications, 2011:590 595.
[4] Haji-Pakir Mohamed Ismail, Shamsuddin Alina Level of Supply Chain Collaboration of Malaysian SME Manufact urers.

[5] Milner, J. M. On the complementary value of accurate demand information and production and supplier flexibility, Manufacturing and Service Operations Management, 2002, 4(2): 99-113.

[6] Raghunathan, S. Impact of demand correlation on the value of and incentives for information sharing in a supply chain, European Journal of Operational Research, 2003, 146: 634-649.

[7] Lin, F., Huang, S. and Lin, S. Effects of information sharing on supply chain performance in electronic commerce, IEEE Transactions on Engineering Management, 2002, 49(3), 258-268. 